\title{
Evaluación de estudiantes universitarios sobre competencias docentes para la acción tutorial
}

\author{
Evaluation of University Students on Teaching Competences for the Tutorial Action \\ Alicia Briones-Olvera ${ }^{a}$
}

\begin{abstract}
:
Evaluate is a useful activity in higher education institutions to make decisions and contribute to educational quality. The objective of the present investigation was to characterize the perception of university students towards the teaching competences for the tutorial action. With a non-experimental design, a cross-sectional study and with a descriptive scope, participated a non-probabilistic, intentional sample of 724 students from Instituto Tecnologico Superior del Oriente (ITESA) of Hidalgo State, who answered online a questionnaire of 20 items (Alfa $=.97)$, with respect to seven competences: empathic attitude, commitment to the tutorial activity, capacity for the tutorial action, disposition to attend the students, ability to guide the students in academic decisions and satisfaction with the attention received. The results, in general, indicate that $85.5 \%$ of students perceive that the tutor competences are good to excellent, while $14.5 \%$ consider them as regular to bad, specifically in the empathic attitude competition the highest percentage was observed $(55 \%)$ in the category of excellent (respect of the tutor towards the group) and the highest percentage in the category of bad $(2.5 \%)$ was for the climate of trust that the tutor achieved. It is concluded that the tutorial teaching competences in ITESA are adequate but some areas of opportunity should be considered. Finally, it is affirmed that evaluation is an appropriate means to assess the processes in favor of integral education that some institutions currently offer.
\end{abstract}

Keywords:

Tutorial action, teaching competences, university students, integral education

\section{Resumen:}

Evaluar es una actividad útil en las instituciones de enseñanza superior para tomar decisiones y coadyuvar en la calidad educativa. El objetivo de la presente investigación fue caracterizar la percepción de los estudiantes universitarios hacia las competencias docentes para la acción tutorial. Con un diseño no experimental, tipo de estudio transversal y con alcance descriptivo, participó una muestra no-probabilística, intencional de 724 estudiantes adscritos al Instituto Tecnológico Superior del Oriente (ITESA) del Estado de Hidalgo, quienes contestaron en línea un cuestionario de 20 preguntas $(A l f a=.97)$, respecto a siete competencias: actitud empática, compromiso con la actividad tutorial, capacidad para la acción tutorial, disposición para atender a los estudiantes, capacidad para orientar a los estudiantes en decisiones académicas y satisfacción con la atención recibida. Los resultados de manera general, indican que $85.5 \%$ de los estudiantes perciben que las competencias de sus tutores son de buenas a excelentes, mientras que $14.5 \%$ las consideran de regulares a malas, específicamente en la competencia de actitud empática, se observó el mayor porcentaje (55\%) en la categoría de excelente (respecto del tutor hacia el grupo) y el mayor porcentaje en la categoría de malo (2.5\%) fue para el clima de confianza que logró el tutor. Se concluye que las competencias docentes tutoriales en el ITESA son adecuadas, pero se deben considerar algunas áreas de oportunidad. Finalmente, se afirma que la evaluación es un medio apropiado para valorar los procesos en pro de la educación integral que brindan actualmente algunas instituciones.

\section{Palabras Clave:}

Acción tutorial, competencias docentes, universitarios, educación integral

\section{INTRODUCCIÓN}

En Educación Superior la tutoría se ha incorporado como una estrategia de acompañamiento y formación integral dirigida a los estudiantes.
Bisquerra (2011) refiere que, para ello es preciso que los docentes desarrollen competencias que les permita ejercer dicha función, apoyándose en teorías y modelos de

intervención educativa, en donde se considere que el tutor es el profesor que orienta, asesora y acompaña al alumno durante su 
estancia en la universidad guiándolo y estimulando la capacidad de hacerse responsable de su propio aprendizaje y de su formación (García, Cuevas, Vales, \& Cruz, 2012).

La tutoría como modelo de intervención, permite tener acercamiento a las necesidades de los alumnos, no sólo las de su proceso de aprendizaje, sino también de las personales y sociales.

No obstante, no debe perder de vista que uno de los retos a los que se enfrentan los estudiantes universitarios es la transición educativa; entendida ésta como el proceso natural que viven los alumnos durante la escolarización derivado de cambios en los niveles educativos, de desarrollo, evolución , crisis y rupturas interpersonales, entre otras.

Por lo que, es imperante que el docente-tutor no sólo domine los contenidos de su profesión, sino que es necesario, que éste adquiera herramientas para planificar acciones que promuevan el desarrollo personal y profesional del estudiante (Aguilar et al., 2015; Álvarez, Moncho, Ramos, Crespo, \& Alonso, 2013).

En este sentido, Castaño, Blanco y Asensio (2012) consideran que la acción tutorial constituye uno de los pilares de la docencia universitaria para realizar el seguimiento académico, la planificación de objetivos de aprendizaje y el apoyo en la adaptación del alumno al entorno académico, lo que confiere al docente un nuevo rol en el que tendrá que desarrollar o entrenarse en competencias para la intervención tutorial como es el caso de la comunicación eficaz, escucha activa, manejo de conflictos, planificación, trabajo individual y en equipo.

\section{DEFINICIÓN DE COMPETENCIAS}

La noción de competencia no proviene ni de un solo paradigma teórico, ni de una sola tradición psicopedagógica, su uso y aplicación se da de acuerdo con las necesidades y orientaciones de cada autor (Guzmán \& Marín, 2011).

Al respecto, Morales, Morales y Bustos (2017), sostienen que la mayoría de las definiciones de competencia profesional no se limita al conjunto de habilidades o destrezas requeridas para desempeñarse adecuadamente en un determinado contexto, ni a la simple ejecución de tareas, sino que también involucra una combinación de atributos con respecto al saber, saber hacer, saber estar y saber ser.

\section{COMPETENCIAS PARA LA ACCIÓN TUTORIAL}

Salazar, Chiang y Muñoz (2016) argumentan que la docencia debe ser ejercida con responsabilidad, ética profesional y con las competencias necesarias para practicarla.

Específicamente, las competencias para la acción tutorial son consideradas como un conjunto de conocimientos, habilidades y actitudes que utilizan los docentes para orientar, acompañar y apoyar a los estudiantes ante las diversas necesidades que se les presentan durante su trayectoria académica, con el objetivo de mejorar la calidad educativa.
Así mismo, Amor y Dios (2017) describen que las competencias para la acción tutorial fomentan la formación integral del alumnado y se orientan en tres pilares de desarrollo:

a) Académico. Se refiere a conocimientos y procesos de aprendizaje.

b) Personal. Referente a actitudes y valores, donde el asesoramiento se centra en la atención al bienestar general del alumno.

c) Profesional. Se refiere a habilidades y destrezas orientadas a la planeación de actividades.

Castaño et al. (2012) afirman que el hecho de adquirir competencias para la intervención tutorial, tiene una doble vertiente, por un lado, se refiere a alcanzar competencias para desempeñarse como tutor y por el otro, aquellas que deberá desarrollar en sus tutorados, estos autores consideran que para el lograr o encaminarse a un proceso adecuado de la acción tutorial, se requiere desarrollar las siguientes cuatro competencias:

1. Comunicación eficaz (escucha activa).

2. Manejo de conflictos.

3. Participación y trabajo en equipo.

4. Planificación del tiempo.

No obstante, Fresán y Romo (2011), tomando como base el Programa Institucional de Tutoría de la Asociación Nacional de Universidades e Instituciones de Educación Superior (ANUIES) describen las siguientes competencias tutoriales:

a) Orientación y apoyo en problemas escolares y/o personales que surgen durante el proceso formativo y en su caso, canalizar a instancias especializadas para su atención.

b) Apoyo al alumno en el proceso de toma de decisiones.

c) Apoyo al alumno en el desarrollo de una metodología de estudio.

d) Seguimiento al alumno con relación a los procesos de aprendizaje y trabajo académico para detectar dificultades y necesidades especiales.

e) Señalar y sugerir actividades extracurriculares para favorecer un desarrollo profesional integral del estudiante.

f) Proporcionar las condiciones para establecer una relación de confianza.

g) Fomentar en los estudiantes el desarrollo de actitudes particulares y de habilidades sociales que faciliten su integración al entorno sociocultural.

h) Estimular en el alumno el conocimiento y aceptación de sí mismo, la construcción de valores, actitudes y hábitos positivos.

i) Proporcionar información académico-administrativo.

j) Brindar o canalizar para atención especializada con asesorías académicas.

PROGRAMA DE TUTORÍA COMO MODELO DE INTERVENCIÓN Y ORIENTACIÓN PSICOPEDAGÓGICA 
Bisquerra y Álvarez (2011) sostienen que los modelos de intervención se basan en estrategias, procesos y procedimientos concretos de actuación, es decir, la intervención debe ser una guía para la acción y clasifica los modelos de orientación en diversas categorías, como se observa en la tabla 1.

\section{Tabla 1}

\section{Clasificación de modelos de orientación psicopedagógica}

\begin{tabular}{|c|c|}
\hline Modelo & Categorías \\
\hline \multirow[t]{2}{*}{ Teórico } & $\begin{array}{l}\text { Formulaciones elaboradas por teóricos que } \\
\text { contribuyeron en las diversas corrientes } \\
\text { psicológicas, por ejemplo: conductismo, } \\
\text { psicoanálisis, humanismo, cognitivo, gestáltico, } \\
\text { etc. }\end{array}$ \\
\hline & $\begin{array}{l}\text { Clínico } \\
\text { Aprendizaje }\end{array}$ \\
\hline \multirow[t]{2}{*}{$\begin{array}{l}\text { De } \\
\text { Intervención }\end{array}$} & $\begin{array}{l}\text { Resultantes de la } \\
\text { combinación de modelos } \\
\text { básicos. }\end{array}$ \\
\hline & Psicopedagógico \\
\hline \multirow{3}{*}{ Organizativos } & Comunidades autónomas \\
\hline & $\begin{array}{l}\text { Países de la Unión } \\
\text { Europea } \\
\text { Americano }\end{array}$ \\
\hline & $\begin{array}{l}\text { Centros educativos } \\
\text { particulares } \\
\text { Equipos sectoriales } \\
\text { Gabinetes privados de } \\
\text { orientación }\end{array}$ \\
\hline
\end{tabular}

Nota. Clasificación adaptada de los modelos de orientación e intervención psicopedagógica (Bisquerra \& Álvarez, 2011).

Por lo tanto, la acción tutorial se puede concebir como un modelo de programas, ya que representa una experiencia de aprendizaje planificada, estructurada, diseñada para satisfacer las necesidades de los estudiantes y/o enriquecer determinadas competencias mediante la orientación (Bisquerra \& Álvarez, 2011).

Desde sus elementos constitutivos dicho modelo de programas se estructura en las siguientes cinco fases:

1. Análisis del contexto para detectar necesidades.

2. Formular objetivos.

3. Planificar actividades.

4. Intervención.

5. Evaluación.

El presente estudio se orienta a la quinta fase (evaluación), esto por parte de la percepción de los estudiantes respecto al desempeño de los tutores, de aquí la importancia de definir el concepto de evaluación.

\section{DEFINICIÓN DE EVALUACIÓN}

La evaluación es un "proceso sistemático, válido y confiable, diseñado intencional y técnicamente, para la obtención de datos, orientada a medir la calidad y los logros de un programa o sistema para posteriormente, encaminarse a la toma de decisiones de mejora, tanto del programa como del personal implicado en el mismo" (Ponce, Olivera, De la Cruz, \& Castillo, 2017, pág.7).

Asimismo, Carballo (1969) concibe a la evaluación como el proceso de recogida de datos, análisis e interpretación de la información obtenida, que va a servir de soporte para la toma de decisiones.

Específicamente, la evaluación de la tutoría se reconoce como una práctica social con implicaciones para la sociedad, las instituciones y los individuos (Luna \& Reyes 2015).

Actualmente, en México los procesos de evaluación y de acreditación de la educación superior son efectuados por diversos organismos especializados, constituidos en marcos de referencia específicos, de criterios, indicadores, estándares, de instrumentos de medición y de estrategias de promoción orientadas a contribuir al aseguramiento de la calidad, así como al logro de la equidad educativa. Estos organismos, se encuentran agrupados, básicamente, en torno a los nueve Comités Interinstitucionales (pares académicos) para la Evaluación de Educación Superior (CIEES), creados en 1991, así como de cerca de 30 organismos acreditadores para las diversas áreas disciplinarias, reconocidos por el Consejo para la Acreditación de la Educación Superior (COPAES), surgido en el año 2000. Por tanto, para lograr o garantizar la operación efectiva y eficaz de un programa de tutoría, es necesario realizar evaluaciones periódicas de sus actores principales. Por lo que, la evaluación hacia los tutores por parte de los alumnos es un área de oportunidad para mejorar la educación.

\section{PERCEPCIÓN DE LOS ESTUDIANTES SOBRE LA ACCIÓN TUTORIAL}

Cruz, Hernández, Ramos, Lara y Rodríguez (2017) definen la percepción como un proceso por el cual los individuos organizan e interpretan sus impresiones sensoriales a fin de darle un significado a su ambiente; por lo tanto, la percepción tutorial es un proceso en el que los estudiantes organizan e interpretan sus impresiones sobre dicha actividad. Es necesario aclarar que percepción no es sinónimo de sensación ya que, una sensación es una experiencia que se vive a partir de un estímulo y es la respuesta a un hecho captado a través de los sentidos. Por el contrario, una percepción es la interpretación de una sensación, que pasa a la estructura cognitiva como un recuerdo imborrable, es de carácter subjetivo e individual. Aquello que es captado por los sentidos adquiere un significado y es clasificado en el cerebro.

Suele decirse que la sensación es lo que precede a la percepción (Zúñiga, 2017).

La percepción de una gestión tutorial de calidad está relacionada con el grado de satisfacción que los jóvenes hayan tenido de 
acuerdo a su experiencia con el trabajo realizado por el tutor (Trujillo, Hurtado, \& Juárez, 2015).

\section{INDICADORES PARA EVALUAR COMPETENCIAS DOCENTES PARA LA ACCIÓN TUTORIAL}

Con fundamento en Ponce et al. (2017) la tutoría supone una interacción estrecha entre los actores principales (tutor y tutorado), por ello, la opinión de los estudiantes sobre la misma, alcanza un valor importante. Para evaluar esta actividad se requieren indicadores propios, diferentes a los utilizados para evaluar la docencia frente a grupo. Tal es el caso de la empatía y el respeto por el individuo, la capacidad para la acción tutorial, el conocimiento de la normatividad institucional, la disposición a atender a los tutorados y la orientación acertada de los estudiantes, son las principales dimensiones a través de las cuales se puede evaluar el desempeño de los tutores (Fresán \& Romo, 2011). En la tabla 2 se muestran las variables e indicadores recomendados para evaluar la acción tutorial.

La evaluación de la percepción de la satisfacción del estudiante con relación a la tutoría universitaria tiene como propósito mejorar el proceso, los avances y resultados de la intervención educativa y a la par, elevar los indicadores de eficiencia en la educación superior. De este modo, evaluar la percepción de los estudiantes sobre la acción tutorial, permitirá además implementar estrategias de mejora del Plan de Acción Tutorial (PAT), así como la gestión del plan de capacitación docente.

Al tomar como base, el marco anterior de referencia, el objetivo de la presente investigación caracterizar la percepción de los estudiantes de nivel superior hacia las competencias de la acción tutorial para identificar fortalezas y debilidades y, de este modo diseñar futuras intervenciones que contribuyan a la mejora de dicha actividad y coadyuvar en la calidad educativa y la formación integral de los estudiantes.

\section{MÉTODO}

\section{DISEÑO, TIPO DE ESTUDIO Y ALCANCES DE INVESTIGACIÓN}

El objeto de estudio de la presente investigación se abordó desde una metodología cuantitativa con un diseño no experimental, transversal y con un alcance descriptivo. Así mismo, el procesamiento y análisis descriptivo de la información se realizó con ayuda del Paquete Estadístico para las Ciencias Sociales (SPSS por sus siglas en inglés) versión 22, disponible en la Web.

\section{PARTICIPANTES Y TIPO DE MUESTREO}

Se trabajó con una muestra no probabilística por conveniencia ya que, la población (alumnos de primer y segundo semestre) recibe acompañamiento tutorial cada semana como parte de su carga académica a diferencia de los demás estudiantes (tercer a octavo semestre) donde el acompañamiento es ocasional.

\section{Tabla 2}

Variables e indicadores para evaluar la percepción de los estudiantes sobre las competencias para la acción tutorial de los docentes

\begin{tabular}{|c|c|}
\hline VARIABLES & INDICADORES \\
\hline $\begin{array}{l}\text { Actitud } \\
\text { empática }\end{array}$ & $\begin{array}{l}\text { Cordialidad y capacidad para crear un clima de } \\
\text { confianza con el estudiante. } \\
\text { Respeto y atención en el trato con el estudiante. }\end{array}$ \\
\hline $\begin{array}{l}\text { Compromiso } \\
\text { con la } \\
\text { actividad } \\
\text { tutorial }\end{array}$ & $\begin{array}{l}\text { Interés en los problemas académicos y personales } \\
\text { que afectan el rendimiento del estudiante. } \\
\text { Capacidad para escuchar los problemas de los } \\
\text { estudiantes. }\end{array}$ \\
\hline $\begin{array}{l}\text { Capacidad } \\
\text { para la } \\
\text { Acción } \\
\text { Tutorial }\end{array}$ & $\begin{array}{l}\text { Capacidad para resolver dudas académicas del } \\
\text { estudiante. } \\
\text { Capacidad para orientar al estudiante en } \\
\text { metodología y técnicas de estudio. } \\
\text { Capacidad para diagnosticar las dificultades y } \\
\text { para realizar las acciones pertinentes para } \\
\text { resolverlas. } \\
\text { Capacidad para estimular el estudio independiente } \\
\text { Formación profesional en su especialidad. } \\
\text { Dominio de métodos pedagógicos para la atención } \\
\text { individualizada o grupal. }\end{array}$ \\
\hline $\begin{array}{l}\text { Disposición } \\
\text { para atender } \\
\text { a los } \\
\text { estudiantes }\end{array}$ & $\begin{array}{l}\text { Disposición a mantener una comunicación } \\
\text { permanente con el estudiante }\end{array}$ \\
\hline $\begin{array}{l}\text { Capacidad } \\
\text { para orientar } \\
\text { a los } \\
\text { estudiantes } \\
\text { en decisiones } \\
\text { académicas }\end{array}$ & $\begin{array}{l}\text { Conocimientos de la normatividad de la } \\
\text { institución en cuanto a los planes de estudio del } \\
\text { nivel de licenciatura. } \\
\text { Orientación en cuanto a la selección de } \\
\text { trayectorias académicas. } \\
\text { Canalización adecuada y oportuna del estudiante } \\
\text { a instancias que le proporcionan una atención } \\
\text { especializada. }\end{array}$ \\
\hline Satisfacción & $\begin{array}{l}\text { Percepción de una influencia positiva de la tutoría } \\
\text { en el desempeño académico. } \\
\text { Percepción de una influencia positiva de la tutoría } \\
\text { en la integración del estudiante a la institución. } \\
\text { Satisfacción del estudiante con el programa de } \\
\text { tutorías. } \\
\text { Satisfacción del estudiante con la actuación del } \\
\text { tutor asignado. }\end{array}$ \\
\hline
\end{tabular}

Nota. Información adaptada de los Programas Institucionales de Tutoría: una propuesta de la ANUIES para su organización y su funcionamiento en las instituciones de educación superior (Fresán \& Romo, 2011).

La muestra la componen 724 estudiantes de primer y segundo semestre, quienes recibieron tutoría durante el ciclo escolar julio-diciembre 2017, correspondientes a los nueve programas educativos que se imparten en el Instituto Tecnológico Superior del Oriente (ITESA). En la tabla 3 se describe el número de estudiantes de acuerdo con el programa educativo. 
Tabla 3

Número de estudiantes que participaron en el estudio de acuerdo al programa educativo.

\begin{tabular}{|c|c|c|}
\hline \multicolumn{2}{|l|}{ Programa Educativo } & $\begin{array}{l}\text { Estudiantes de primer } \\
\text { y segundo semestre }\end{array}$ \\
\hline \multicolumn{2}{|l|}{ Ingeniería Civil } & 86 \\
\hline \multicolumn{2}{|c|}{ Ingeniería Electromecánica } & 18 \\
\hline \multicolumn{2}{|c|}{ Ingeniería en Gestión Empresarial } & 31 \\
\hline $\begin{array}{l}\text { Ingeniería en } \\
\text { Alimentarias } \\
\text { Ingeniería en Logísti }\end{array}$ & Industrias & 23 \\
\hline \multicolumn{2}{|c|}{ Ingeniería Mecatrónica } & 42 \\
\hline $\begin{array}{l}\text { Ingeniería en } \\
\text { Automotrices }\end{array}$ & Sistemas & 104 \\
\hline $\begin{array}{l}\text { Ingeniería en } \\
\text { Computacionales }\end{array}$ & Sistemas & 36 \\
\hline \multicolumn{2}{|c|}{$\begin{array}{l}\text { Computacionales } \\
\text { Licenciatura en Administración }\end{array}$} & 42 \\
\hline Total: & & 724 \\
\hline
\end{tabular}

Del total (724) de los participantes, 298 son mujeres $(41.2 \%)$ y (426) son hombres (58.8\%), sus edades oscilan entre los 16 y 40 años de edad.

\section{ESCENARIO DE EVALUACIÓN}

La evaluación se llevó a cabo en las instalaciones del ITESA, el cual se encuentra ubicado en la región del altiplano, específicamente, en el municipio de Apan, Hidalgo.

El ITESA, ha sido evaluado por organismos acreditadores como el CIEES, obteniendo la certificación de sus programas educativos con nivel 1 , por lo que se considera una institución que ofrece educación de calidad.

La plantilla del personal docente está conformado por 108 docentes, de los cuáles actualmente 19 (17.5\%) se desempeñan como tutores que atienden a estudiantes de primer y segundo semestre de forma regular.

\section{INSTRUMENTO DE EVALUACIÓN}

La percepción de los estudiantes sobre la acción tutorial se recabó a través de un cuestionario en línea que se instaló en el Portal de Tutoría del ITESA. El cuestionario se basa en las cinco competencias que debe poseer un tutor, las cuales son propuestas por los programas estandarizados de la ANUIES: actitud empática, compromiso con la actividad tutorial, capacidad para la acción tutorial, disposición para atender a los estudiantes, capacidad para orientar a los estudiantes en decisiones académicas y satisfacción con la atención recibida. Se trata de una escala tipo Likert, la cual consta de 20 preguntas con opción múltiple de respuesta, donde 1 significa malo, 2 deficiente, 3 regular, 4 bueno y 5 excelente. En la tabla 4, se describe el número de reactivos para cada una de las competencias evaluadas. El tiempo de respuesta es de 15 minutos aproximadamente. Para esta investigación el cuestionario posee una confiabilidad elevada $($ Alfa $=.97)$.

\section{Tabla 4}

Competencia y número de reactivos del cuestionario de evaluación de la acción tutorial

\begin{tabular}{lc}
\hline Competencia Evaluada & $\begin{array}{c}\text { Número de } \\
\text { reactivo }\end{array}$ \\
\hline $\begin{array}{l}\text { Satisfacción } \\
\text { Actitud empática }\end{array}$ & $1,3,4,16,19,20$ \\
$\begin{array}{l}\text { Compromiso con la actividad } \\
\text { tutorial }\end{array}$ & $6,7,17$ \\
$\begin{array}{l}\text { Disposición para atender a los } \\
\text { estudiantes } \\
\text { Capacidad para orientar a los } \\
\text { estudiantes en decisiones } \\
\text { académicas para la acción } \\
\begin{array}{l}\text { Capacidad pad } \\
\text { tutorial }\end{array}\end{array}$ & 8,12 \\
\hline
\end{tabular}

\section{PROCEDIMIENTO}

La evaluación se realizó al finalizar el semestre julio-diciembre 2017, durante un periodo de dos semanas, en los casos en los que los estudiantes no asistieron el día de la evaluación, se les solicitó mediante su correo institucional contestar el cuestionario, por tanto, se obtuvo una tasa de respuesta del 100\%. Cada estudiante ingresó al portal con su matrícula y una vez registrados, aparecía el consentimiento informado y las instrucciones, haciendo énfasis en el anonimato de los datos, con el fin de recaban información veraz.

Los resultados fueron dados a conocer a cada docente mediante un oficio con la calificación obtenida en cada indicador, así como recomendaciones de mejora para incorporar en su plan de acción tutorial.

\section{RESULTADOS}

Sabiendo que la práctica docente debe adecuarse al contexto y sobre todo a las necesidades de los estudiantes para lograr la calidad educativa, es importante identificar o conocer las opiniones y percepciones que tienen acerca de la acción tutorial. En la tabla 5 se caracteriza la percepción de los estudiantes hacia los tutores, esto en frecuencias y porcentajes de acuerdo a las cinco categorías que evalúa el cuestionario. Como puede observarse, de manera general, un alto porcentaje de los estudiantes percibe de buena a excelente la acción tutorial $(85.5 \%)$, mientras que $14.5 \%$ la consideran de regular a mala. 
Tabla 5

\section{Caracterización de la percepción de los alumnos hacia la} acción tutorial $(\mathrm{N}=724)$

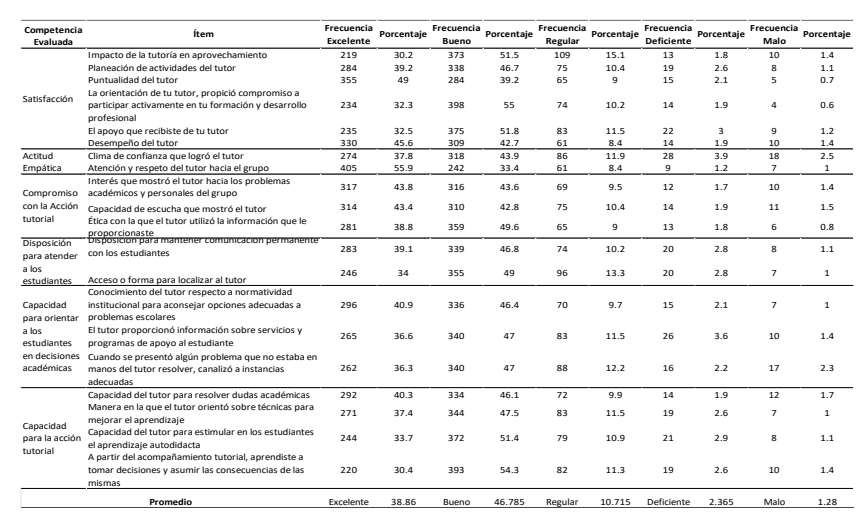

\section{Satisfacción}

Específicamente, en cuanto a la satisfacción, $49.0 \%$ de estudiantes evaluaron como excelente la puntualidad y $45.6 \%$ consideran excelente el desempeño del tutor.

\section{Actitud empática}

Destaca que, de las siete competencias evaluadas, la de actitud empática, fue la que obtuvo el mayor porcentaje (55.9\%) en la categoría de excelente, concretamente para el indicador "atención y respeto que mostró el tutor hacia el grupo". En tanto que, el mayor porcentaje $(2.5 \%)$ para la categoría de malo, se observó para el "clima de confianza que logró el tutor".

\section{Compromiso con la acción tutorial}

Respecto al compromiso con la acción tutorial, 43.8\% de estudiantes calificó como excelente el interés que el tutor muestra hacia los problemas académicos y personales del grupo y $43.4 \%$ evalúa como excelente la capacidad de escucha.

\section{Disposición para atender a los estudiantes}

Con relación a la disposición del tutor para atender a los estudiantes, destaca que $49.0 \%$ calificó como bueno el acceso o forma de localizar al tutor.

\section{Capacidad para orientar a los estudiantes en decisiones académicas}

En cuanto a la capacidad que poseen los tutores para orientar a los estudiantes en decisiones académicas, $47.0 \%$ de los estudiantes califica como buena la información que les proporcionó el tutor, aunque existe oportunidad de mejora con respecto a la canalización a instancias adecuadas cuando el problema sale de las manos del docente.

\section{Capacidad para la acción tutorial}

En cuanto a la acción tutorial en general, $54.3 \%$ de estudiantes calificó como bueno el haber aprendido a partir de la orientación de su tutor para tomar decisiones y asumir las consecuencias de las mismas, mientras que $51.4 \%$ calificó como bueno la capacidad del tutor para estimular en los estudiantes el aprendizaje autodidacta.

\section{DISCUSIÓN}

Evaluar es una actividad útil en las instituciones de enseñanza superior para tomar decisiones y coadyuvar en la calidad educativa, tanto del desempeño de los tutores como del proceso enseñanza-aprendizaje (Joao, Sotolongo, \& Martínez, 2016).

Los hallazgos de esta investigación están en línea con investigaciones previas, ya que, los alumnos perciben el programa de tutoría de bueno a excelente, por lo que, es sustancial, que el tutor establezca un clima de confianza, los trate con respeto y esté dispuesto a escucharlos (Castaño et al., Ponce et al., 2017; Villarreal, Nava, \& Urdiales, 2015).

Sin embargo, es necesario implementar estrategias para mejorar los indicadores - que, aunque su porcentaje fue bajo- en la opción de regular, deficiente o malo, es necesario hacer énfasis en crear un clima de confianza y canalizar a instancias adecuadas cuando se presenta algún problema que no está en manos del tutor resolver. Por tanto, ésta es un área de oportunidad que puede implementarse con una capacitación a los tutores y mejorar la acción tutorial.

Otra estrategia sería contemplar las siguientes actividades a realizar con los estudiantes en los siguientes momentos: a) previo al ingreso a la universidad para identificar necesidades de orientación e información, b) al inicio de los estudios universitarios para generar acciones que generen confianza, motivación, adaptación e integración a la vida universitaria, c) durante los estudios universitarios para reforzar estrategias de enseñanza-aprendizaje y d) al finalizar los estudios universitarios para apoyar en la inserción al ámbito laboral (Álvarez, 2015).

En el caso de la canalización a instancias adecuadas, se sugiere establecer redes de apoyo con las cuales se puedan remitir a los estudiantes que requieran de atención especializada o algún servicio que la misma institución u otras ofrecen como: atención psicológica, servicio médico, becas, asesorías académicas, etcétera y de esta forma mantener comunicación y seguimiento con las áreas correspondientes.

El realizar la canalización a instancias especializadas, permitirá brindar al estudiante la orientación oportuna que les facilite la toma de decisiones apropiada, evitando así la deserción y de acuerdo a las necesidades específicas de desarrollo personal, profesional o académico. 
La evaluación de la satisfacción de los estudiantes respecto a la tutoría en resumen, tiene como objetivo ir encaminada a la introducción de elementos de mejora o de buenas prácticas en los Planes de Acción Tutorial (Pérez, Martínez, \& Martínez, 2015; Trujillo et al., 2015). Por lo que los datos aquí mostrados cumplen el objetivo general de la investigación.

\section{CONCLUSIÓN}

Sin duda, evaluar es un recurso que permite mejorar la calidad de cualquier servicio y el académico, no es la excepción. Se concluye que la percepción de la acción tutorial de manera general en el ITESA es de buena a excelente. De manera específica, la información obtenida permite observar que la satisfacción, la actitud empática, el compromiso y las competencias con la acción tutorial, la disposición para atender a los estudiantes, capacidad para orientar en decisiones académicas, así como la capacidad para la acción tutorial de los docentes del ITESA de acuerdo con la percepción de los estudiantes es de buena a excelente. Sólo un porcentaje pequeño percibe la acción tutorial como regular, deficiente o mala, por lo que se deben desarrollar estrategias para mejorar dicha acción tutorial. Se afirma que la evaluación es un medio apropiado para valorar los procesos en pro de la educación integral que brindan actualmente algunas instituciones.

Finalmente, se deben considerar que los hallazgos no se pueden generalizar a la población, ya que se trató de una muestra no probabilística de tipo intencional. Futuras investigaciones deberán realizar una evaluación más amplia que permita fortalecer estos hallazgos, así como contrastar los datos de acuerdo al programa académico y de acuerdo al semestre.

\section{AGRADECIMIENTOS}

La autora agradece al Dr. Rubén García Cruz, al Mtro. José Antonio Zamora Guido y a la Mtra. Nidia Irais Moreno Vargas por su guía en la elaboración del presente artículo. Asimismo, agradece a las autoridades del Instituto Tecnológico Superior del Oriente del Estado de Hidalgo por las facilidades otorgadas para la realización de la investigación.

\section{REFERENCIAS}

Aguilar, J. M., Alías, A., Álvarez, J., Fernández, J.M., Pérez, E.R., \& Hernández, A.I. (2015). Necesidades de formación del profesor universitario en competencias relacionadas con la acción tutorial. Revista de Docencia Universitaria, 13(3), 357-375. Recuperado de https://polipapers.upv.es/index.php/REDU/article/view/5433

Álvarez, M. \& Álvarez, J. (2015). La tutoría universitaria: del modo actual a un modelo integral. Revista Electrónica Interuniversitaria de Formación del Profesorado, 18(2), 125-142. doi: http://dx.doi.org/10.6018/reifop.18.2.219671

Amor, M. I. \& Dios, I. (2017). La tutoría universitaria: un espacio para la orientación personal, académica y profesional en la formación inicial del profesorado. Revista Española de Orientación y Psicopedagogía, 28(1), 119-130. Doi: 10.5944/reop.vol.28.num.1.2017.19362
Bisquerra, R. (2011). Modelos de orientación e intervención psicopedagógica. Madrid: Wolters Kluwer España, S.A.

Caballo, R. (1996). Evaluación de intervención tutorial. Revista Complutense de Educación, 7(1), 97-118.

Castaño, E., Blanco, A., \& Asensio, E. (2012). Competencias para la tutoría: experiencia de formación con profesores universitarios. Revista de Docencia Universitaria, 10(2), 194-2010. Recuperado de https://polipapers.upv.es/index.php/REDU/article/view/6103

Cruz, F., Hernández, I., Ramos, I., Lara, C., \& Rodríguez, D.L. (2017). Percepción de los estudiantes de enfermería sobre las tutorías académicas. Revista Electrónica de la Coordinación Universitaria de Observatorios de la Universidad Veracruzana, 3, 84-89. Recuperado de https://www.uv.mx/uvserva/articulos/percepcion-de-los-estudiantesde-enfermeria-sobre-las-tutorias-academicas/

Fresan, M. \& Romo, A. (2011). Programas institucionales de tutoría: una propuesta de la ANUIES. México: Colección documentos ANUIES.

García, R., Cuevas, O., Vales, J. J., \& Cruz, I.R. (2012). Impacto del programa de tutoría en el desempeño académico de los alumnos del Instituto Tecnológico de Sonora. Revista Electrónica de Investigación Educativa, 14(1), 106-121. Recuperado de https://redie.uabc.mx/redie/article/viewFile/299/462

Guzmán, I. \& Marín, R. (2011). La competencia y las competencias docentes: reflexiones sobre el concepto y la evaluación. Revista Electrónica Interuniversitaria de Formación del Profesorado, 14(1), 151-163. Recuperado de https://www.aufop.com/aufop/uploaded_files/articulos/1301588498.pd f

Joao, D., Sotolongo, M., \& Martínez, C. C. (2016). Procedimiento para la evaluación del desempeño del personal docente en las universidades públicas angolanas. Ingeniería Industrial, 37(2), 178-189. Recuperado de https://www.redalyc.org/html/3604/360446197007/

Luna, E. \& Reyes, E.P (2015). Validación de constructo de un cuestionario de evaluación de la competencia docente. Revista Electrónica de Investigación Educativa, 17(3), 13-27. Recuperado de https://redie.uabc.mx/redie/article/view/1090/1291

Morales, R., Morales, R., \& Bustos M. (2017). Competencias docentes y desempeño: un estudio con profesores universitarios. Revista Publicando, 4(1), 248-269. Recuperado de https://revistapublicando.org/revista/index.php/crv/article/view/522

Pérez, F. J., Martínez, P., \& Martínez, M. (2015). Satisfacción del estudiante universitario con la tutoría. Diseño y validación de un instrumento de medida. Estudios Sobre Educación, 29(22), 81-101.

Ponce, L., Olivera, M.E., De la Cruz, A., \& Castillo, C.M. (2017). Evaluación de la acción tutorial en la facultad de contaduría pública. Revista Electrónica sobre Educación Media y Superior, 4(7), 1-17. Recuperado http://www.cemys.org.mx/index.php/CEMYS/article/view/273

Romo, A. \& Romero, A. (2015). Cómo mejorar la visión de los programas acreditadores mexicanos, sobre la importancia de la acción tutorial. Revista Electrónica Interuniversitaria de Formación del Profesorado, 18(2), 145-159. Recuperado de https://dialnet.unirioja.es/servlet/articulo?codigo $=5097276$

Salazar, C. M., Chiang, M. M., \& Muñoz, Y. A. (2016). Competencias docentes en la Educación Superior: Un estudio empírico en la Universidad del Bio-Bio. Revista Electrónica Actualidades Investigativas en Educación, 6(1), 1-28. Recuperado de http://www.scielo.sa.cr/pdf/aie/v16n1/1409-4703-aie-16-01-00253.pdf

Trujillo, A., Hurtado, O., \& Juárez, C. (2015). Percepciones de los estudiantes sobre desempeño del tutor en el plantel Lic. Adolfo López Mateos, de la Universidad Autónoma del Estado de México: una aproximación desde el pensamiento complejo. Revista Dilemas Contemporáneos: Educación, Política y Valores, 3(4), 1-17. Recuperado de https://www.dilemascontemporaneoseducacionpoliticayvalores.com/ 
Villareal, M. G., Nava, A. I, \& Urdiales, M. E. (2015). Percepción de estudiantes de psicología sobre la actividad tutorial. Repositorio Académico Digital. Recuperado de http://eprints.uanl.mx/8471/

Zúñiga, J. R. (2017). Percepción del sistema de tutoría universitaria, según los estudiantes del X semestre de las escuelas profesionales de la Facultad de Ciencias y Tecnologías Sociales y Humanidades (Tesis de Maestría). Universidad Católica de Santa María, Perú. 